\title{
Atoms in Gaseous and Solid States and their Energy and Force Relationships under Transitional Behaviors
}

Mubarak Ali ( $\square$ mubarak74@mail.com )

COMSATS University Islamabad https://orcid.org/0000-0003-1612-6014

\section{Physical Sciences - Article}

Keywords: Atomic structure, Atomic state, Overt photons, Energy knots, Transition energy, Orientation force

Posted Date: December 19th, 2020

DOI: https://doi.org/10.21203/rs.3.rs-88120/v2

License: (c) (1) This work is licensed under a Creative Commons Attribution 4.0 International License.

Read Full License 


\section{Abstract}

Recalling the conventional insights of different atomic states, it is possible to discover new insights, which can cope with the existing challenges. Atoms, in fact, form from the electrons and energy knot nets. Suitably intercrossed overt photons construct energy knots in atoms of all elements. In growing atoms of gaseous and solid states, schemes of intercrossing overt photons become different. To construct atomic lattice in any element, overt photons in suitable length and number intercross by keeping the centers of their lengths at a common point. A scheme of intercrossing overt photons frames energy knots simultaneously clamping to positioned electrons. Atoms are differentiated on the basis of their different numbers of energy knots and electrons. A number of unfilled states in an atom represents a valency. Excluding hydrogen, atoms possess the same valency as specified for them. However, two more electrons with two already prescribed ones for the first shell form the zeroth ring of atom. In the hydrogen atom, only two electrons are occupied by two energy knots; two overt photons of the least measured lengths intercross to form the shape like digit eight. In this way, four electrons remain occupied by four energy knots to form helium atom. Thus, a helium atom is related to a zeroth ring in all higher order atoms. In order to validate these aforementioned statements, the concept of studying protons and neutrons is no longer significant. As far as the atoms of gaseous state are concerned, electrons possess minimum required potential energy. In this way, electrons of gaseous atoms remain above the middle of occupied energy knots in more than half the length, and they keep on experiencing maximum required levitational force along the north pole. In atoms of solid state, electrons possess maximum required potential energy. In this way, electrons of solid atoms remain below the middle of occupied energy knots in more than half the length, and they keep on experiencing maximum required gravitational force along the south pole. Each transition state of the atom is under the established relation of energy and force. Under transitional energy of an atom, electrons deal with infinitesimal displacements within their occupied energy knots, where the orientational force keeps on engaging them to introduce the recovery, neutral, re-crystallization and liquid states. Electrons left to the center of atom orientate from north to east clockwise, and electrons right to the centre of atom orientate from north to west anti-clockwise during the conversion of gaseous atom to liquid state. On the other hand, electrons left to the center of atom orientate from south to east anti-clockwise, and electrons right to the center of atom orientate from south to west clockwise during the conversion of solid atom to liquid state. These fundamental revolutions shed new light on the development of sustainable science and engineering.

\section{Introduction}

Understanding the mechanisms of atomic formation and then relating them with each other help to develop sustainable science behind the technologically important materials. The Periodic Table shows the position of atoms in various elements in the form of rows and columns by referring to their characteristics based on atomic number, mass number, valence number, electronic configuration, atomic radius, electronegativity and shielding effect, etc. The Periodic Table also provides information of filled 
and unfilled states of electrons, where valency of the atom is assigned. However, filled and unfilled states in atoms of different elements are in different ways.

The lattice (energy-knot-net) of carbon atom is the same for different allotropes, but each allotrope has different position of filled and unfilled states [1]. Solid atoms belonging to different elements elongate at an appropriate level of ground surface [2]. The developing mechanism of various tiny-sized particles has been explained, where gold atoms possess different behaviors of the electronic structure [3]. Transitional atoms of gold element gather to form a monolayer assembly at the solution surface, where triangleshaped tiny particles are developed under the supplied nanoenergy packets [4]. Structural evolutions of atoms executing confined interstate electron dynamics involve conservative forces, which have been discussed in a separate study [5]. The phenomena of heat and photon energy have been explored, where electron dynamics of the silicon atom convert heat energy into photon energy [6]. These studies indicate that atoms of different elements exhibit different electronic structures of the existing ones.

Nevertheless, mercury belongs to transition metals group, where it neither shows solid state nor gaseous state, but it behaves like liquid. Metals such as cesium, gallium and rubidium remain in solid state at room temperature, and start to melt above the room temperature. These specify that filled and unfilled states of the outer ring play an important role in originating the characteristics of atoms. Further, inert gas atoms do not show any sort of affinity with atoms of other elements because of inertness. In this way, they do not bind to evolve the structure; they split under the excessive propagation of photons having characteristics of the photonic current [2]. Therefore, elongation or deformation of solid atoms and splitting of inert gas atoms indicate different behaviors of atoms. In this study, a basic relation of energy and force in different-natured atoms dealing with respective transition states is explored.

At suitable concentration of gold precursor, a large number of tiny-sized particles got developed having a triangular shape [7]. Morphological structure of gold particles was developed through different bipolar and unipolar pulses [8]. In tiny-metallic colloids, the incompatible packing developed the particles of distorted shapes and compatible packing developed the particles of geometrical shapes [9]. In the pulsebased process, the processing of gold solution developed particles of geometrical shapes and the processing of silver solution did not develop the particles of geometrical shapes [10]. Particles of unprecedented shapes were developed, thereby identifying the specific role of energy and force in the process of developing [11]. The use of tiny-sized particles for nanomedicine can be either effective or defective because of the varying behavior of comprised atoms [12]. These studies deduce a different behavior of atoms to the existing one.

Atomic structure of different carbon allotropes along with their binding has been discussed [1]. Different results of testing and analysis from different regions of the deposited carbon film explain that how difficult it is to reach for an appropriate conclusion [13]. Morphology and structure of particles in depositing carbon films altered under the variation of localized process conditions [14]. Carbon films developed under different morphologies and structures of grains and particles because of having different chamber pressures [15]. 
The possibility of assembling colloidal matter into meaningful structure enables atoms and molecules to be candidates for future materials [16]. Understanding the individual dynamics of formation of tiny-sized particles is essential prior to their assembling into useful large-sized particles [17]. Hard coating is due to the varyingly switched energy and forced behaviors of gaseous and solid atoms, where non-conservative energy is engaged at the first stage [18].

Sir Isaac Newton formulated the laws of motion and universal gravitation. The law of universal gravitation involves the mathematical description of gravity. Sir Albert Einstein developed a general theory of relativity along with mass and energy relationship and the principle of relativity was further explained by extending it to the gravitational field, where the concept of anti-gravity (levity) was not incited; this general theory of relativity remained only a model to a large-scale spectrum structure. The different models such as Rutherford's atomic model and Bohr's atomic model are available in the literature defining atomic structure. In addition to these, Yukawa's theory explains the stability of nucleus (neutron to neutron binding) in an atom.

Solid atoms are eligible to evolve different structures when they treat below the suitable level of ground surface. Their ground points also initiate binding below the ground surface. Solid atoms under transitional behavior are eligible to develop different structures when the exerting forces to electrons are in the surface format $[3,4,7-11]$. Based on these observations, the structure evolution in solid atoms has been discussed, where interstate electron dynamics of atoms involved conservative forces [5]. This indicates again a different behavior of atoms instead of the existing ones describing shells, orbits, band gap, fermi levels, nucleus, etc.

The previous electronic structures describe the atoms with respect to orbital configurations and shells. In the latter one, the nucleus of an atom also describes protons and neutrons. Quite often, the electronic structures describe atoms with the quantized states in recent works, but the science of materials raises a fundamental question that how these atoms form. Why do atoms exist in gaseous and solid states? What kind of descriptive mechanism do they require in their formation? Orientation force exerting at the electron level should depend on the atomic state, i.e., gaseous, semisolid or solid. In this way, atoms of different elements should deal with different levels of force. Accordingly, atoms of different elements should deal with different levels of energy, so a generalized relationship between energy and force is also presented here when atoms undertake their transition states.

\section{Results And Discussion}

Depending on the attained dynamics and transition state, atoms develop different tiny-sized particles [3]. To develop a mono layer tiny-shaped particle, solid atoms gather in the compact monolayer assembly [4]. A structure evolution in atoms of the suitable elements has been discussed, where confined interstate electron dynamics involves conservative forces [5]. At different chamber pressures, carbon films were synthesized having discernable features of their morphology and structure [15]. Incompatible working 
energy and forced behaviors of gaseous and solid atoms develop hard coating [18]. These studies show that atoms possess different energy and force behaviors.

Atomic structure of the carbon atom in different allotropic forms was discussed [1]; a lattice or energyknot-net in each allotropic form of the carbon atom remained the same. A lattice of carbon atom constructed by the overt photons having suitable length and number was also discussed in the study. Overt photons are the subsets of the main stream photons [6]. Atoms do not ionize; they modify to elongate or deform under the solid state; they split under the inert state and they squeeze under the gaseous state [2]. This indicates that the centre of an atom of any element does not involve mass of the electron, so the centre of atom should be at the point of intercrossed overt photons.

In the formation of lattice belonging to any atom, overt photons having appropriate lengths and numbers intercrossed by keeping the centre of their lengths at a common point. The force and energy of intercrossed overt photons should remain actual, constructing energy knots of filled and unfilled states of the atom.

Overt photons in suitable lengths design the filled and unfilled states of different atoms. Overt photons construct filled and unfilled states of energy knots as per the requirement of the atoms. In this way, atoms of different elements differentiate from each other. Intercrossing overt photons to construct lattice of the atom (in any element) is in such a manner that energy knots clamp positioned electrons. In the atoms of gaseous, semisolid and solid elements, the scheme of intercrossing overt photons becomes different.

Excluding hydrogen atom, addition of two more electrons in the central ring of any atom is required to form the zeroth ring. Atoms are already known to have first shell, which has occupied two electrons. However, the first shell is a zeroth ring, which needs four electrons in the present case. Therefore, an atom requires two more electrons to form the zeroth ring. The zeroth ring can be termed as nucleus. When no electron is available for empty energy knot, it is referred to as unfilled state. The number of unfilled state indicates the valency of atom. When the surface force is exerted to the electrons of solid atom at the appropriate level of ground surface, energy knots clamped electrons are stretched along both sides (eastwest poles) from the centre [2].

Atoms consist of electrons clamping having sizeable energy knots. Excluding the atoms of inert behavior elements, atoms of different elements also possess the unfilled states. A least measured length overt photon is formed by the length of two 'unit photons', where each unit photon has a shape like 'Gaussian distribution of turned ends'. When two least measured length overt photons intercross, they construct a knot through intercrossing. Hence, a shape of a tilted digit 'eight' is formed, which is related to the lattice of hydrogen atom. The intercrossing of two shapes of tilted digit 'eight' forms the molecular hydrogen, where number of electrons becomes equal to number of electrons in helium atom. However, a helium atom contains four electrons under the originally built-in lattice of energy knots instead of separately intercrossed two shapes of tilted digit 'eight'. In the lattice of helium atom, two shapes of digit 'eight' are formed on intercrossing four overt photons having the least measured lengths. 
Following the zeroth ring, atoms contain either first ring, second ring and so on. In this way, arrangement of electrons in the available rings (of different atoms) other than the zeroth rings is in the same manner as previously mentioned. Nevertheless, two more electrons are added to shape the zeroth ring in atoms of all elements except hydrogen. In addition to two more filled states in the zeroth ring, a net of energy knots in atom of any element follows the same description of filled and unfilled states as mentioned previously except hydrogen element. In atoms of various elements other than hydrogen element, the central four filled state electrons form the zeroth ring.

Two overt photons of the least measured length form a tilted digit 'eight' as shown in Figure 1 (a). The electronic configuration of hydrogen atom, hydrogen molecule and helium atom are displayed in Figure 1 (b), (c) and (d), respectively. When two photons of the least measured lengths intercrossed, they form a shape of tilted digit 'eight', which is the lattice of hydrogen atom as shown in Figure 1 (a). Electrons of the tiniest mass trapped in the empty spaces formed by the energy knots (black and green ones) are shown in Figure 1 (b). Two hydrogen atoms overlap to form the molecular hydrogen as shown in Figure 1 (c). The structure of helium atom is shown in Figure 1 (d).

Atoms of some elements can keep empty spaces left at the outer ends of constituted chains. In an atom, terminated ends of chains are related to the outer ring. An empty space is exactly the size of electron. For example, an argon atom might have eight empty spaces in the outer ring in addition to eight filled states as indicated by the arrows in Figure 2. These eight empty spaces might not be related to unfilled states. Here, to build shorter chains of states for each case, intercrossed overt photons construct a chain of states having length which is short by a unit photon at both ends. The presented observation justifies the sketched model of argon atom displayed in Figure 2.

The structure of lithium atom is shown in Figure 3. The zeroth ring is related to nucleus. The outer ring is related to the first ring, which is also displayed in Figure 3. In Figure 3, lithium atom has a large volume to store energy as arrowed in the regions labelled by 1,2,3 and 4 . Due to this capacity of storing energy, the structure of lithium is considered quite suitable for energy storage. The lithium atom contains two chains of states as labeled in Figure 3.

An atom describing valency involves both filled and unfilled states in the outer ring. However to execute interstate electron dynamics, either non-confined [1] or confined [5], an atom requires a suitable position of its filled and unfilled states in the outer ring. Inert gas atoms neither undertake confined nor nonconfined electron dynamics. Further investigation is required to understand the nature of atoms belonging to elements of inert behavior. A carbon atom remains in gaseous state, semisolid state or solid state depending on the position of electrons and unfilled states in the outer ring. By changing the position of an electron in the nearby suitable unfilled state, a carbon atom gets converted into another state [1]. The present unfilled states or empty energy knots in the outer rings of different atoms are according to the prescribed numbers of electrons and valency. In hydrogen atom, one more electron is required to fill the second state of electron. The hydrogen atom does not contain the zeroth ring due to the presence of 
two electrons in total. The zeroth ring of an atom in all elements can be termed as nucleus. In this way, helium atom is only related to the zeroth ring having no further ring.

The centre of the atom is located at the center of length of each intercrossed overt photon. Therefore, the electrons have more than half of the mass (length) to the upward sides (along the north pole) from the mid of the occupied energy knots, and atoms behave in the gaseous state. In the gaseous atom, energy knots clamped electrons undertake contraction as per potential energy of the electrons. Nonetheless, the electrons have more than half of the mass (length) to the downward sides (along the south pole) from the mid of the occupied energy knots, and atoms behave in the solid state. In the solid atom, energy knots clamped electrons undertake stretching as per potential energy of the electrons.

In the original gaseous state, atom keeps the ground point in space format by obeying the original energy and force. When an atom is converted from gaseous state to liquid state, it gains transitional energy $\left(E_{T}\right)$, increasing the level of energy. Electrons decrease the levitational force $\left(F_{L}\right)$ exerting along the relevant poles. In this way, electrons of the atom increase potential energy to undertake liquid state, so ground point of the atom reaches near the ground surface. If the liquid state atom gets restored to the original gaseous state, the gained ' $\mathrm{E}_{\mathrm{T}}$ ' is released. (By occupying the energy knots, electrons can tilt to upward under the self-restoration, so the ' $\mathrm{E}_{\mathrm{T}}$ ' is in the absorbing manner.) In this way, atom achieves the original state under required increasing ' $F_{L}$ ' exerting along the relevant poles of electrons. This is an inversely proportional relationship of ' $\mathrm{E}_{\mathrm{T}}$ ' and ' $\mathrm{F}_{\mathrm{L}}$ '. A generalized relationship in gaseous atoms is shown in Figure 4 (a).

In the conversion of a gaseous atom from original state to liquid state, ' $\mathrm{E}_{\mathrm{T}}$ ' is gained by the atom which is inversely proportional to engaging ' $F_{\mathrm{L}}$ ' exerted at the electron level and is indicated in equation (1).

$E_{T}$ a $1 / F_{L}$ or $E_{T}=L_{e} \times 1 / F_{L} \ldots(1)$

Electrons of the gaseous atoms deal with the low potential energy. ' $\mathrm{L}_{e}$ ' indicates the number of electrons in a gaseous atom. ' $\mathrm{L}_{e}$ ' is different for atoms of different gaseous elements, but, ' $\mathrm{L}_{e}$ ' is constant for atoms of the same element in equation (1). The chemical activity of transitional gaseous atoms introduces different chemical reactivity. Both energy and force behaviors change in the transition state of the gaseous atom.

When an atom of solid behavior is converted into liquid state, ' $\mathrm{E}_{\mathrm{T}}$ ' absorbs positioned electrons in the required orientation. Thus, electrons minimize potential energy dealing with solid atom in negative functioning. A gravitational force $\left(\mathrm{F}_{\mathrm{G}}\right)$ exerting along the relevant poles of electrons decrease, so tilting of the electrons is upward. Electrons of the atom taking liquid state minimize potential energy to tilt upward under infinitesimal displacements. In the course of taking infinitesimal displacements, electrons remain within their occupied energy knots. When the liquid behavior atom gets restored to the original (solid) behavior, the equal amount of energy in the sense of gaining manner is involved to attain original ground 
point. Thus, atom deals with the positive work. Hence, the solid atoms dealing with the liquid states is in a direct relationship between ' $\mathrm{E}_{\mathrm{T}}$ ' and ' $\mathrm{F}_{\mathrm{G}}$ ' as sketched in Figure 4 (b).

In the conversion of a solid atom from original state to liquid state, ' $\mathrm{E}_{\mathrm{T}}$ ' absorbed the atom and is directly proportional to engaging ' $\mathrm{F}_{\mathrm{G}}$ ' exerting at the electron level as indicated in equation (2).

$E_{T}$ a $F_{G}$ or $E_{T}=G_{e} \times F_{G} \ldots(2)$

Electrons of solid atoms deal with high potential energy. ' $G_{e}$ ' indicates the number of electrons in a solid atom. ' $G$ ' is different for atoms of different solid elements. Thus, ' $\mathrm{G}_{\mathrm{e}}$ ' is constant for atoms of the same element in equation (2). Chemical activity of transitional solid atoms introduces different chemical reactivity. Energy and force behaviors are changed in each established transitional behavior of the solid atom.

In Figure 5, the electrons of the hypothesized gaseous atom depict different transitional behaviors as per tilting: only left-positioned and right-positioned electron to the centre of atom are considered. In the original gaseous state atom, left-positioned electron keeps orientation along $40^{\circ}$, which is on the left side to normal line drawn from the center as shown in Figure 5 (a); right-positioned electron also keeps orientation along $40^{\circ}$, which is on the right side to normal line drawn from the center. For the recovery state, left-positioned electron keeps orientation along $20^{\circ}$, which is on the left side to normal line drawn from the center as shown in Figure 5 (b); right-positioned electron also keeps orientation along $20^{\circ}$, which is on the right side to normal line drawn from the center. For the neutral state, left-positioned electron keeps orientation along $5^{\circ}$, which is on the left side to normal line drawn from the center as shown in Figure 5 (c); right-positioned electron also keeps orientation along $5^{\circ}$, which is on the right side to normal line drawn from the center. For the re-crystallization and liquid states, left-positioned electrons keep orientations along $25^{\circ}$ and $50^{\circ}$, respectively, which are on the right sides to normal lines drawn from the centers as shown in Figure 5 (d) and Figure 5 (e), respectively; right-positioned electrons also keep orientations along $25^{\circ}$ and $50^{\circ}$, respectively, which are on the left sides to normal lines drawn from the centers. Degrees related to orientations of electrons are in approximate number.

In Figure 6, the electrons of the hypothesized solid atom depict transitional behaviors as per tilting; only left-positioned and right-positioned electron to the centre of atom are considered. Electrons of the solid atoms deal with the same degree of orientation undertaking different transition states as in the case of gaseous atoms, but along the south poles as discussed in the section of Supplementary Information.

Electrons do cross projected lines from the normal lines to the centers in the course of re-crystallization and liquid states; however, they do not cross projected poles of atoms. This is shown in Figure 5 for the case of gaseous atom and in Figure 6 for the case of solid atom. The centers of the hypothesized gaseous atom and solid atom are also shown in Figures 5 and 6. Poles (axes) of left-positioned and rightpositioned electrons (to the center of atom) in both hypothesized gaseous atom and solid atom for neutral states are labeled in Figure 5 and Figure 6, respectively. In both hypothesized gaseous and solid 
atoms, the origin of reference of left-positioned electron (to the center of atom) is different from the origin of reference of right-positioned electron (to the center of atom). In the original gaseous atom and solid atom, both left-positioned and right-positioned electrons (to the center of atom) keep orientating towards the north from the upward sides and towards the south from the downward sides, respectively. In both gaseous and solid atoms, electrons change the features of occupied energy knots depending on the orientational force and potential energy. In Figures 5 and 6 , electrons do not show clamping energy knots and the tilting in different transition states is symbolically shown by the curved arrows.

A left-positioned electron and right-positioned electron to the centre of hypothesized gaseous atom deal with clockwise and anti-clockwise tilting, respectively, while undertaking different transition states. A leftpositioned electron and right-positioned electron to the centre of hypothesized solid atom deal with anticlockwise and clockwise tilting, respectively, while undertaking different transition states. Transitional behaviors of the atoms are being controlled from the centers. Different orientations of electrons resulted under the correspondence of external environment are also being accommodated from the centers of the atoms. In gaseous and solid atoms, a zone related to the exerting impartial force at the electron level is discussed elsewhere [5].

In an atom, a state of electron is related to unfilled state and a state of valency is related to unfilled state. States of electrons (filled states) and valency (unfilled states) are referred to atoms of gaseous, semisolid and solid states in the same way. However, nets of energy knots constructed by the intercrossing of overt photons in atoms of gaseous states clamp electrons from the downward sides. In the atoms of solid states, nets of energy knots are constructed by the intercrossing of overt photons clamp electrons from the upward sides. Hence, in the atoms of semisolid states, nets of energy knots are constructed by the intercrossing of overt photons clamp electrons of laterally-orientated position from the centers (mid). Therefore, the formation of schemes of lattices in atoms of gaseous, semisolid and solid states is different, but atoms keep the conserved amounts of force and energy in the original format of ground points - gaseous atoms in the space format, semisolid atoms in the surface format, and solid atoms in the grounded format.

In the transition state, either in gaseous atom or in solid atom, electrons deal with infinitesimal displacements by remaining within the occupied energy knots. For this reason, the relation of energy and force in atoms of gaseous and solid states has been discussed above. A gaseous or solid atom undergoes liquid state by varying the potential energy of comprised electrons, where electrons remain clamped by the respective energy knots. For gaseous atoms in liquid state, infinitesimal displacements of electrons undergo to downward sides, where the lengths (of electrons) become nearly halfway to mid of the occupied energy knots. For solid atoms in liquid state, infinitesimal displacements of electrons undergo to upward sides, where the lengths (of electrons) also become nearly halfway to mid of the occupied energy knots.

The formation of atoms of different elements is in the zones allocated for them. An electron is not discussed in the context of negative charge, but it is discussed as a particle. The particles of smallest 
sizes make the electronic structure of atom. An electron of any atom is the smallest unit of concrete mass. It forms the basis of atom in terms of the exerting forces, i.e., gravitation, levitation and surface.

In the formation of certain natured atoms, some of the energy knots neither work for filled states nor for unfilled states, which remain folded by neighboring chains of energy knots. Folded energy knots in different chains are shown in the atomic structure of titanium [18]. The trapping and capturing of pieces of electron are particularly in the regions of zeroth rings belonging to atoms of suitable elements. Many overt photons intercrossed (by keeping centers at a common point) under a particular scheme to construct a required number of chains (of states) in an atom. However, only four electrons (of complete shape and size) in an atom are eligible to settle in the zeroth ring. Particles of the fractional sizes of an electron may be captured by the folded energy knots not working for filled states and unfilled states in certain behavior atoms. This kind of work can be studied in different branches of physics. The broken pieces of matter though smaller in size to electron can further diversify particle physics and neutrino physics.

Electrons of suitable atoms undertake (non-stop) infinitesimal displacements within the occupied energy knots (where atoms do not deal with elongation or deformation); they can generate radiations of different types rather than photons. (When the required amount of heat energy was available, a unit photon is generated by the forward direction cycle or reverse direction cycle of confined interstate electron dynamics of silicon atom [6].) When solid atoms deal with the transition states and electrons remain restricted by the occupied energy knots under the heat energy of tits and bits, they cannot deal with the elastically-driven electronic states. In this way under plastically-driven electronic states, solid atoms keep elongation or deformation originating the science of condensed matter [19]. This is also the case in arrays of solid atoms as they convert into the structures of smooth elements [4].

To clamp the tiniest masses called electrons, the net of energy knots is required to form an atom as discussed above. Formation of the highly purified form of matter in the smallest shape is an unprecedented phenomenon of nature. Formation of the tiniest matter and nets of energy knots to shape atoms requires a suitable environment, so the formation of atoms locates a suitable environment to reveal the features. The characteristics of atoms for each element are different, which can be categorized in already named classes such as gaseous atoms, semisolid atoms and solid atoms. Hence, atoms of any element grow in their respective environment. Naturally, this is according to the conditions that are required to grow atoms of a distinct nature. Construction of energy knots and their clamping to electrons are one of the most extra-ordinary processes. Hence, atoms of different elements grow at suitable places or zones of the exerting forces.

Atoms of gaseous states grow in their respective environment which can be in different zones of space, so astronomers, environmentalists, chemists, space scientists and those working in the allied areas can look into the nature of growing atoms. Atoms of semisolid states grow at ground level, so electrical engineers, earth scientists, physicists, environmentalists and those working in the allied areas can look into the nature of growing atoms. Atoms of solid behaviors grow below the ground surface, so 
metallurgists, geologists, chemical engineers, chemists, paleontologists and those working in the allied areas can look into the nature of growing atoms.

Different behaviors of atoms discussed here offer new insights. Atoms start functioning for the possible transition states while dealing with the varying energy and force. The presented scheme of atoms allows one to develop atoms with different lattices and electrons, so it works for a new diversity of matter. This study deals with the general discussions, such as incremental changes that depend on the schemes of intercrossing photons, filled and unfilled states, orientation force, potential energy of electrons and distribution of the electrons in atom. Hence, there is a room for further discussion and investigation that may lead to attest sustainable utilization of resources.

\section{Conclusion}

The formation mechanisms of atoms in different states disclose here. Atomic structure of different elements is elucidated. In transitional gaseous atoms and solid atoms, relationships between energy and force are also explained.

Electrons of the tiniest mass are occupied by the energy knots. Energy knots are constructed by the intercrossing of overt photons while keeping the centers of their lengths at a common point. Energy knots of filled and unfilled states (and some filled states only) of different atoms get constructed by the intercrossing of overt photons under a particular scheme. The schemes of intercrossing overt photons construct states of electrons and unfilled state(s) in different manner in atoms of gaseous and solid states. It is also the case in semisolid atoms. In the scheme of gaseous atoms or solid atoms, atoms specify different number of filled and unfilled states. The number of intercrossing overt photons having particular lengths are according to the number of electrons and valency that an atom keeps for the element.

In the original state of an atom, energy knots clamped electrons keep them in the states. In the intercrossing of overt photons, the element of force remains dressed up by the energy to design a lattice of any atom. In addition to the prescribed number of electrons of different atoms, the addition of two more electrons in the central rings form their zeroth rings. Except hydrogen, atoms of all elements possess two additional electrons along with the already designated two electrons. In this way, four electrons form the zeroth ring altogether in the new scheme of an atom. Hence, an atom does not require protons and neutrons to define the nucleus. At the place of first shell, the first ring is studied. Instead of orbits, shells or quanta, atoms form the zeroth ring, and number of rings keep filled and unfilled states.

The shape of the zeroth ring is like a 'cross', where two shapes of 'eight' digit intercross to keep four electrons. An atomic structure of helium is identical to the zeroth ring, which exists at the place of nucleus in atoms of all elements except hydrogen atom. The zeroth ring in an atom is related to the central ring. In hydrogen atom, one more electron is required along with the one previously designated. However, it does not form the zeroth ring. The structure of hydrogen atom is half to the structure of zeroth ring or helium atom. The shape of digit 'eight' indicates the lattice of hydrogen atom. By filling two electrons in hollow 
spaces of digit 'eight', hydrogen atom is formed. The overlying two hydrogen atoms form a hydrogen molecule, but not the way helium atom forms a structure. Helium atom itself is related to the zeroth ring.

In gaseous atoms, electrons keep more than half of their length above the mid of occupied energy knots along the north poles. Atoms in gaseous state possess minimum required potential energy, so energy knots clamped electrons deal with maximum required contraction. In solid atoms, electrons keep more than half of their length below the mid of occupied energy knots along the south poles. Solid atoms possess maximum required potential energy, so energy knots clamped electrons deal with maximum required stretch.

On supplying transition energy, gaseous and solid atoms undertake transition states, where their electrons deal with infinitesimal displacement by remaining within the occupied energy knots. To undertake liquid states, electrons (by remaining within the occupied energy knots) deal with infinitesimal displacements to the downward and to the upward, respectively in gaseous and solid atoms. Gaseous atoms undertaking transition states engaged levitational force and involved transitional energy in the form of gaining; they function in an inversely proportional relationship. In solid atoms undertaking transition states, engaged gravitational force and involved transitional energy in the form of absorption function in a directly proportional relationship.

Estimated orientations of electrons in gaseous atoms while undertaking original, recovery and neutral states are along $40^{\circ}, 20^{\circ}$ and $5^{\circ}$, respectively. These orientations are from the right sides to normal lines drawn from the centers of right-positioned electrons, and from the left sides to normal lines drawn from the centers of left-positioned electrons. Orientations of electrons are along the north poles. Estimated orientations of electrons in solid atoms while undertaking original, recovery and neutral states are also along $40^{\circ}, 20^{\circ}$ and $5^{\circ}$, respectively. However, orientations of electrons are along the south poles.

Estimated orientations of electrons in gaseous atoms undertaking re-crystallization and liquid states are along $25^{\circ}$ and $50^{\circ}$, respectively. Estimated orientations of electrons in solid atoms undertaking recrystallization and liquid states are also along $25^{\circ}$ and $50^{\circ}$, respectively. (In re-crystallization and liquid states of gaseous or solid atom, orientations are from the opposite sides to normal lines drawn from the centers of electrons.)

The transition of atom is not usually in the environment of original ground point. Thus, an atom undertakes transition under suitable energy. Due to different energy and force behaviors, gaseous and solid atoms deal with different chemical reactivity. Chemical reactivity changes for each transition state of gaseous and solid atoms.

\section{References}

1. M. Ali, Atomic Structure and Binding of Carbon Atoms. https://www.preprints.org/manuscript/201801.0036/v10 
2. M. Ali, Atoms of None of the Elements Ionize While Atoms of Inert Behavior Split by Photonic Current. (2020), http://arxiv.org/abs/1611.05392 (last version)

3. M. Ali, I-N. Lin, Effects of the Electronic Structure, Phase Transition and Localized Dynamics of Atoms in the Formation of Tiny Particles of Gold. (2020), http://arxiv.org/abs/1604.07144 (last version)

4. M. Ali, Tiny-Shaped Particles Developing a Mono Layer Shape Dealing with Localized Gravity and Levity at the Solution Surface. (2020), http://arxiv.org/abs/1609.08047 (last version)

5. M. Ali, Structure Evolutions in Atoms of the Elements Executing Confined Interstate Electron Dynamics. (2020), http://arxiv.org/abs/1611.01255 (last version)

6. M. Ali, Heat and Photon Energy Phenomena: Dealing with Matter at Atomic and Electronic Level. https://www.preprints.org/manuscript/201701.0028/v10

7. M. Ali, I-N. Lin, Development of Gold Tiny Particles and Particles in Different Sizes at Varying Precursor Concentration. Adv. Nat. Sci.: Nanosci. Nanotechnol. 11 (2020) 015006.

8. M. Ali, I-N. Lin, Controlling morphology-structure of gold tiny particles, nanoparticles and particles at different pulse rates and pulse polarity. Adv. Nat. Sci.: Nanosci. Nanotechnol. 10 (2019) 025015.

9. M. Ali, I-N. Lin, Formation of tiny particles and their extended shapes: origin of physics and chemistry of materials. Appl. Nanosci. 9 (2019) 1367-1382.

10. M. Ali, I-N. Lin, C. -J. Yeh. Tapping Opportunity of Tiny-Shaped Particles and Role of Precursor in Developing Shaped Particles. NANO 13 (2018) 1850073 (16 pages).

11. M. Ali, I -N. Lin, C. -J. Yeh, Predictor Packing in Developing Unprecedented Shaped Colloidal Particles. NANO 13 (2018) 1850109 (15 pages).

12. M. Ali, Nanoparticles-Photons: Effective or Defective Nanomedicine. J. Nanomed. Res. 5 (2017) 00139.

13. M. Ali, I-N. Lin, Phase transitions and critical phenomena of tiny grains carbon films synthesized in microwave-based vapor deposition system. Surf. Interface Anal. 51 (2019) 389-399.

14. M. Ali, M. Ürgen, Switching dynamics of morphology-structure in chemically deposited carbon films -A new insight. Carbon 122 (2017) 653-663.

15. M. Ali, M. Ürgen, Morphology and Structure of Carbon Films Deposited at Varying Chamber Pressures. (2020), https://arxiv.org/abs/1802.00730 (last version)

16. S. C. Glotzer, M. J. Solomon, Anisotropy of building blocks and their assembly into complex structures. Nature Mater. 6 (2007) 557-562.

17. S. Link, M. A. El-Sayed, Shape and size dependence of radiative, nonradiative and photothermal properties of gold nanocrystals. Int. Rev. Phys. Chem. 19 (2000) 409- 453.

18. M. Ali, E. Hamzah, M. R. M. Toff, Hard Coating Deposits: Incompatible Working Energy and Forced Behaviors of Gaseous and Solid Atoms. Adv. Mater. Process. Technol. (2020), https://doi.org/10.1080/2374068X.2020.1822055 
19. M. Ali, Transition Energy, Orientation Force and Work Done in Transitional Behavior Atoms:

Formulating New Principles in Thermodynamics. ChemRxiv, Preprint. (2020),

https://doi.org/10.26434/chemrxiv.11553057 (last version) (and also at

https://www.researchgate.net/publication/338485763)

20. M. Ali, I-N. Lin, Gold Nanostructures and Microstructures with Tunable Aspect Ratios for High-Speed Uni- and Multidirectional Photonic Applications. ACS Appl. Nano Mater. 3 (9) (2020) 9410-9424.

\section{Figures}

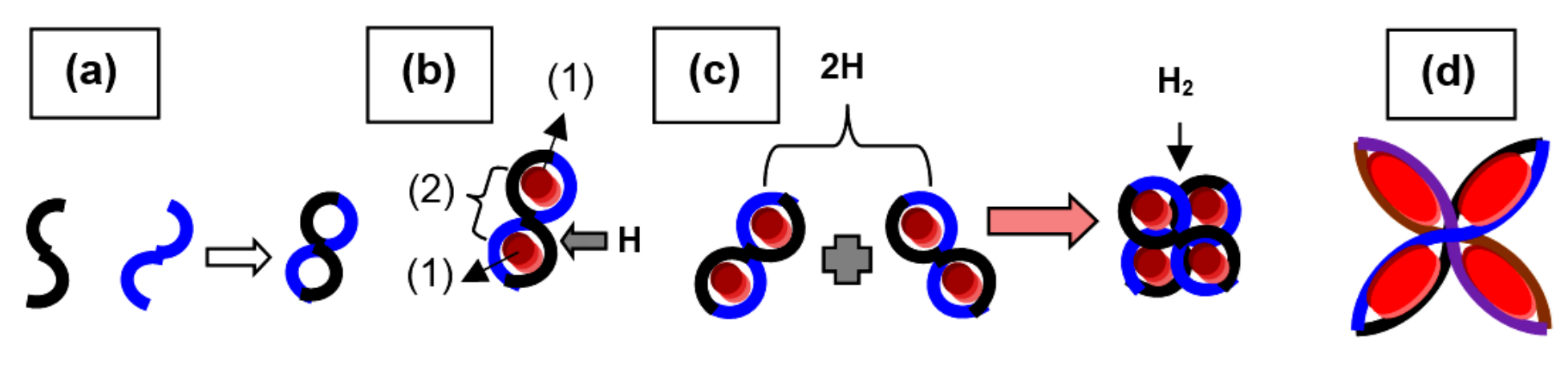

Figure 1

(a) Formation of energy knot net in $\mathrm{H}$ atom (two minimum length overt photons intercrossed to form tilted digit 'eight'), (b) the structure of $\mathrm{H}$ atom showing (1) electrons and (2) occupied energy knots, (c) the formation of $\mathrm{H} 2$ molecule and (d) the structure of helium atom

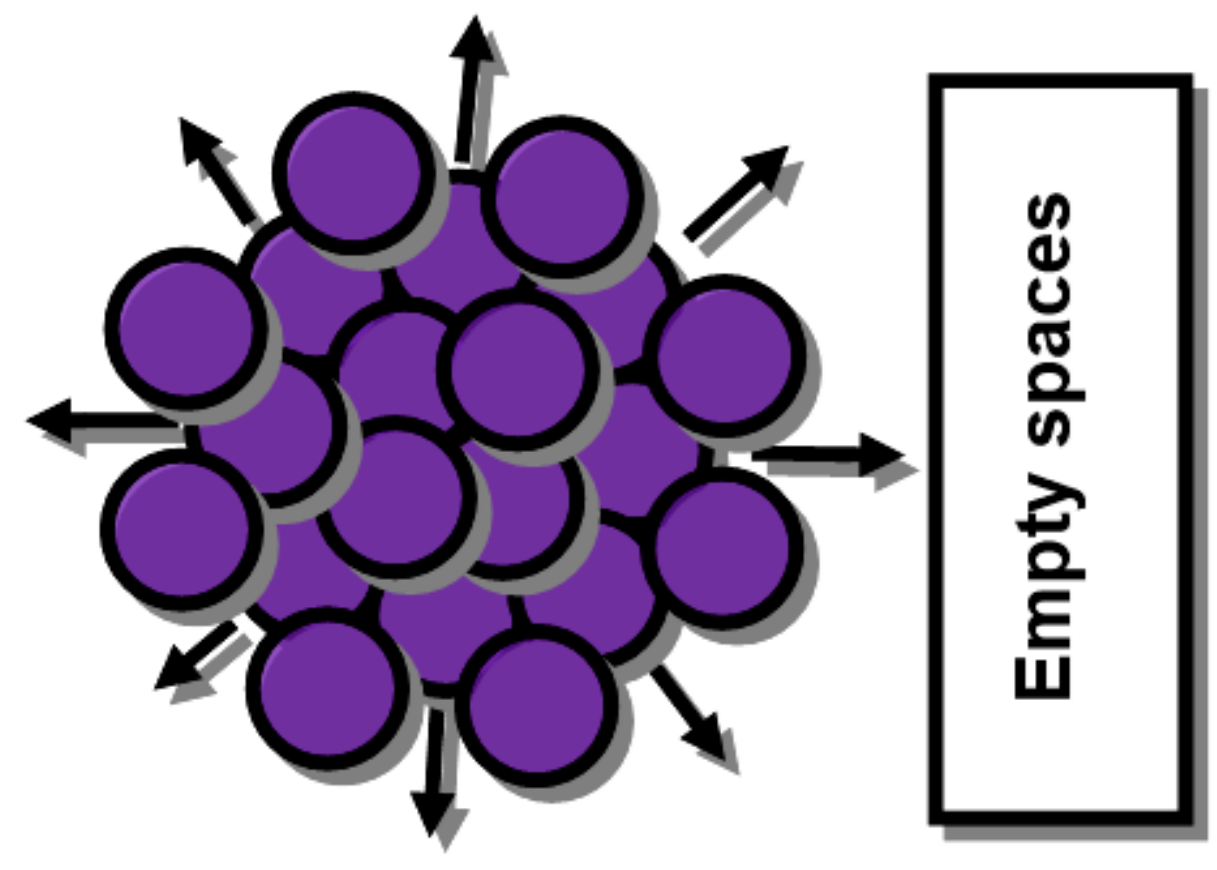

Figure 2

Empty spaces in argon atom indicated by the arrows having length short by a unit photon at both ends of the constituted chains 


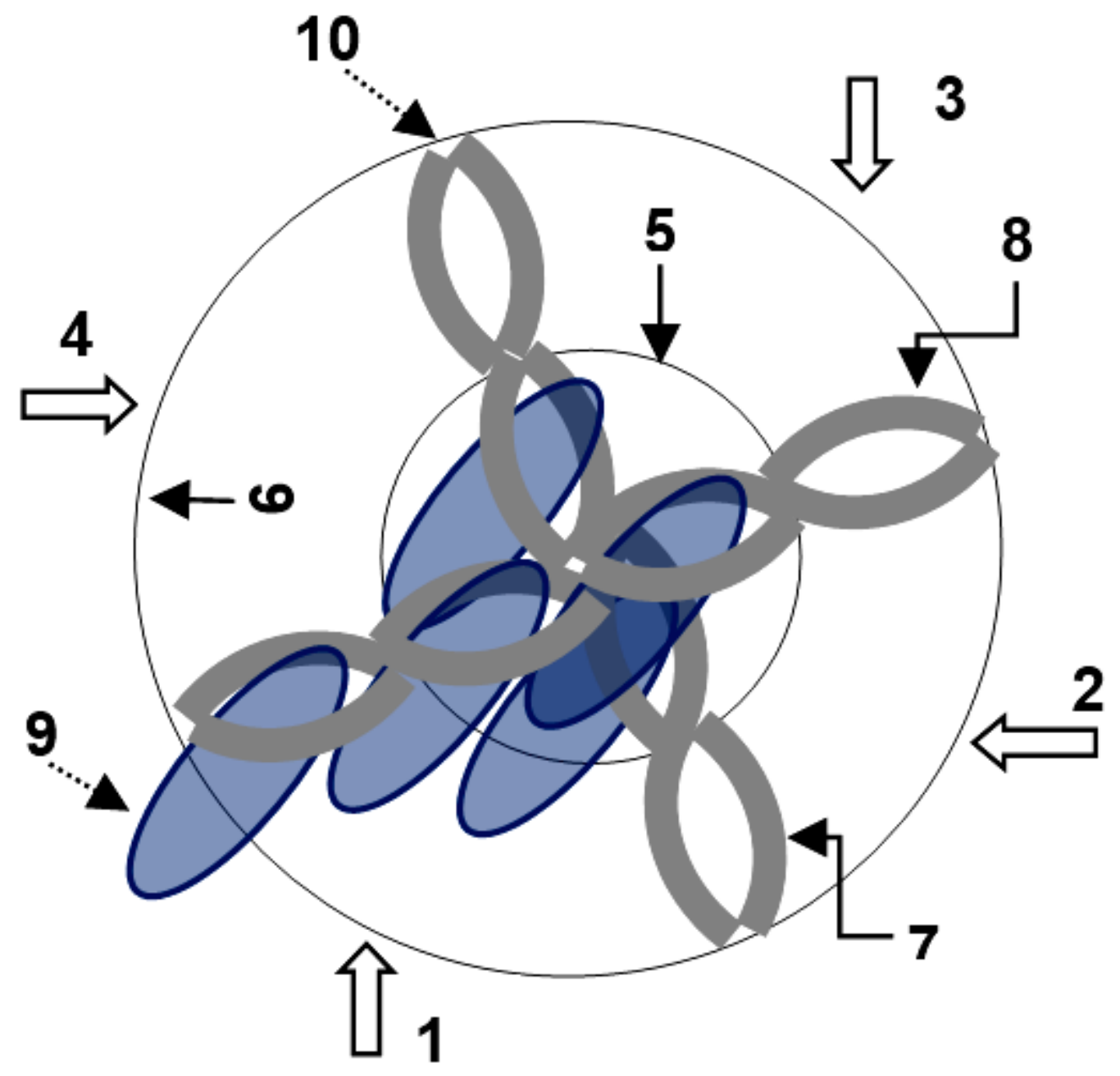

Figure 3

Atomic structure of lithium; 1, 2, 3 \& 4 - energy storage regions, 5 - zeroth ring, 6 - outer ring, 7 \& 8 chains of states, 9 - filled state electron and 10 - unfilled state or empty energy knot
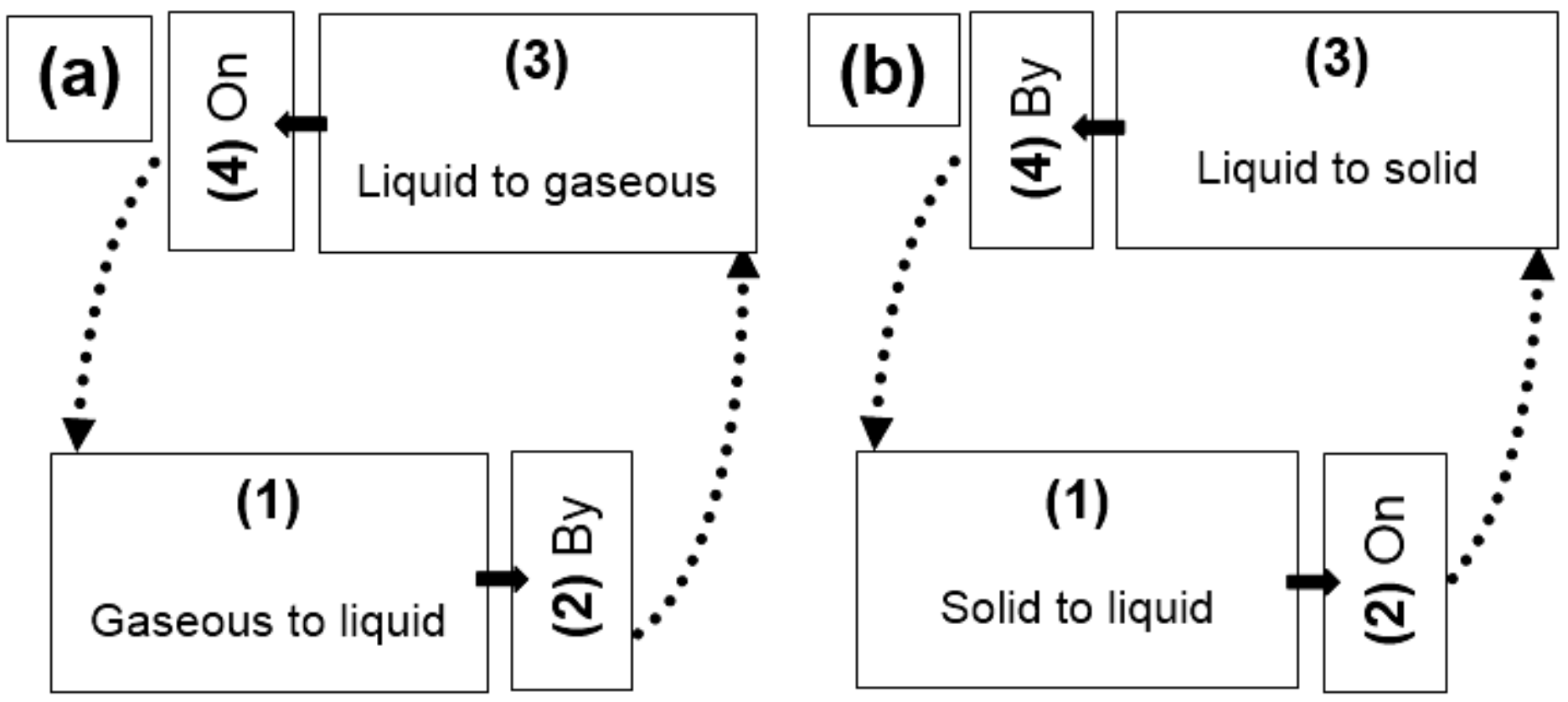


\section{Figure 4}

Generalized energy and force relationship of atoms: (a) Gaseous state atoms convert into the liquid states, and liquid states restore into the gaseous state atoms; (1) conversion of original gaseous state atoms into liquid states, where decreasing ' $F L$ ' is exerted to the electrons (ground points of the atoms reach near the ground surface); (2) work is done by the gaseous atoms; (3) conversion of liquid state atoms into original gaseous states, where increasing 'FL' is exerted to the electrons (ground points of the atoms reach above the ground surface); (4) work is done on the liquid atoms. (b) Solid state atoms convert into the liquid states, and liquid states restore into the solid state atoms: (1) conversion of original solid state atoms into liquid state, where decreasing ' $F G$ ' is exerted to the electrons (ground points of the atoms reach near the ground surface); (2) work is done on the solid atoms; (3) conversion of liquid state atoms into original solid state, where increasing " $F G$ ' is exerted to the electrons (ground points of the atoms reach below the ground surface); (4) work is done by the liquid atoms

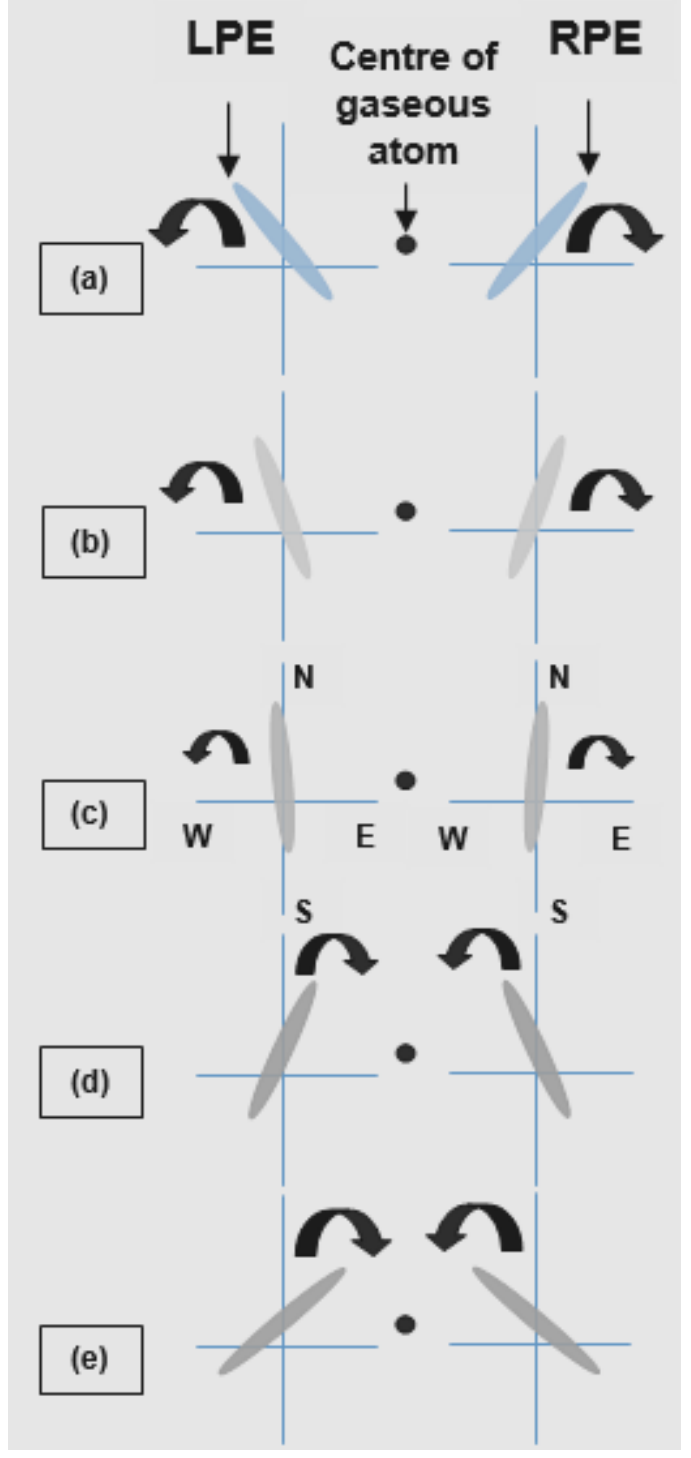

Figure 5 
left-positioned electron (LPE) and right-positioned electron (RPE) to the centre of the hypothesized gaseous atom orientated from the north side (a) along $40^{\circ}$ left to normal line and along $40^{\circ}$ right to normal line in original state, respectively, (b) along $20^{\circ}$ left to normal line and along $20^{\circ}$ right to normal line in recovery state, respectively, (c) along $5^{\circ}$ left to normal line and along $5^{\circ}$ right to normal line in neutral state, respectively, (d) along $25^{\circ}$ right to normal line and along $25^{\circ}$ left to normal line in recrystallization state, respectively, and (e) along $50^{\circ}$ right to normal line and along $50^{\circ}$ left to normal line in liquid state, respectively

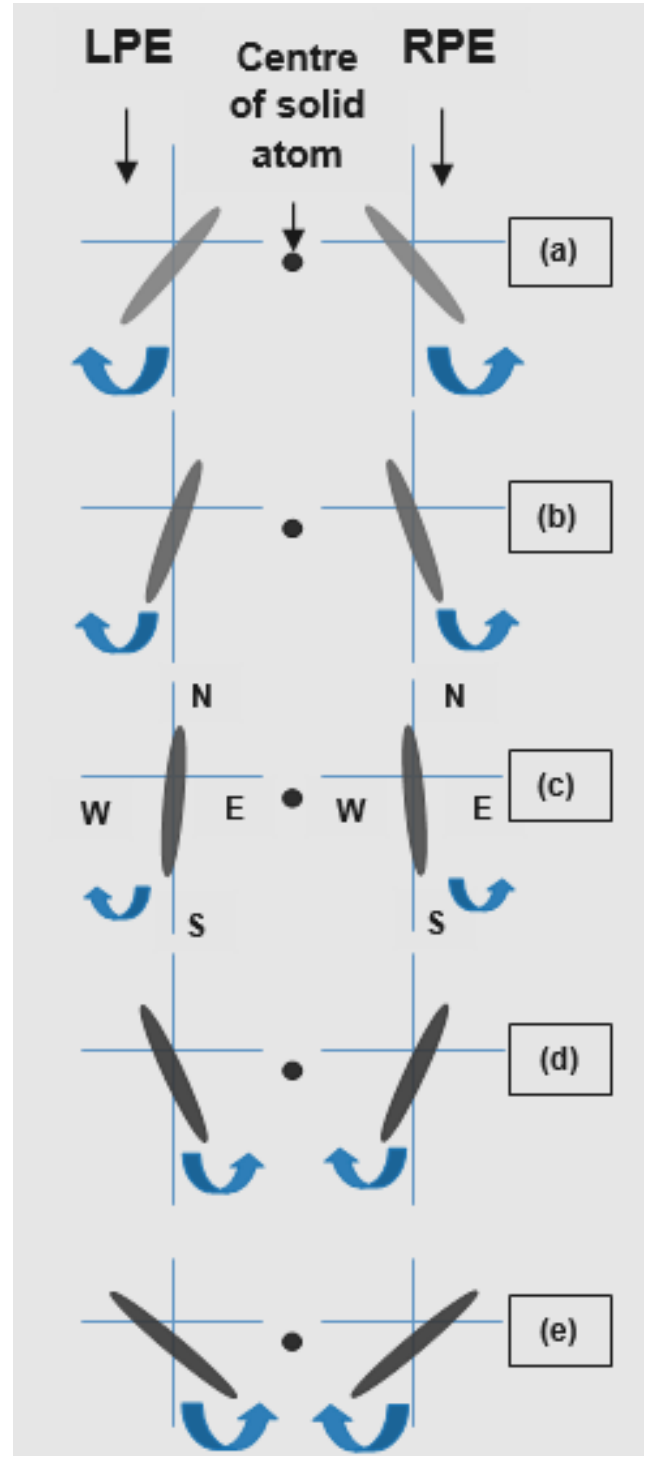

\section{Figure 6}

left-positioned electron (LPE) and right-positioned electron (RPE) to the centre of the hypothesized solid atom orientated from the south side (a) along $40^{\circ}$ left to normal line and along $40^{\circ}$ right to normal line in original state, respectively, (b) along $20^{\circ}$ left to normal line and along $20^{\circ}$ right to normal line in recovery state, respectively, (c) along $5^{\circ}$ left to normal line and along $5^{\circ}$ right to normal line in neutral state, respectively, (d) along $25^{\circ}$ right to normal line and along $25^{\circ}$ left to normal line in re-crystallization state, respectively, and (e) along $50^{\circ}$ right to normal line and along $50^{\circ}$ left to normal line in liquid state, respectively 


\section{Supplementary Files}

This is a list of supplementary files associated with this preprint. Click to download.

- Supplement.Information.pdf 